Abstract-Fish species that are protogynous hermaphrodites generally have sex ratios skewed toward males at large sizes. These skewed sex ratios at length can make a stock more vulnerable to overexploitation, particularly in fisheries where minimum size is regulated, resulting in the removal of reproductively active males. We developed a length-based population model to test the vulnerability to exploitation of a population with an atypical protogynous life history, in particular the northern stock of black sea bass (Centropristis striata). Black sea bass north of Cape Hatteras, North Carolina, are unusual for protogynous species in that they may undergo prematurational transformation, remain female at large sizes, involve secondary males in spawning, and undertake seasonal migrations. The model was developed to examine the impact of participation by secondary males in population productivity, the influence of size at sex transformation, and the subsequent robustness of the population under exploitation, in comparison with equivalent gonochoristic and typical protogynous populations. Although the model does not capture all the dynamics of a population, such as density-dependent regulation of sex transformation, our results indicate that the northern stock of black sea bass may be more resilient in response to exploitation than would be expected if they were typical protogynous hermaphrodites.

Manuscript submitted 21 May 2015. Manuscript accepted 19 August 2016. Fish. Bull. 114:476-489 (2016).

Online publication date: 8 September 2016 . doi: 10.7755/FB.114.4.9

The views and opinions expressed or implied in this article are those of the author (or authors) and do not necessarily reflect the position of the National Marine Fisheries Service, NOAA.

\title{
Evaluating the vulnerability of an atypical protogynous hermaphrodite to fishery exploitation: results from a population model for black sea bass (Centropristis striata)
}

\author{
Jessica Blaylock ${ }^{1}$ \\ Gary R. Shepherd ${ }^{2}$ \\ Email address for contact author: jessica.blaylock@noaa.gov \\ 1 Integrated Statistics \\ 16 Sumner Street \\ Woods Hole, MA 02543 \\ Present address: 3825 South Drive \\ Fort Worth, Texas 76109 \\ 2 Northeast Fisheries Science Center \\ National Marine Fisheries Service, NOAA \\ 166 Water Street \\ Woods Hole, Massachusetts 02543
}

The capacity of a fish population to persist under exploitation is highly dependent on the life history of a species and its ability to effectively reproduce. In gonochoristic species, individuals remain the same sex throughout their lives, generally ensuring a constant presence of both males and females capable of reproduction, even under relatively strong exploitation. In contrast, species with a hermaphroditic life history (either protogynous or protandrous) might not have an adequate abundance of sexually mature individuals across sizes and ages (Provost and Jensen ${ }^{1}$ ). In protogynous species, a common pathway for sex change involves individuals initially maturing and reproducing as female and then

${ }^{1}$ Provost, M., and O. Jensen. 2013. Use of sex change considerations in stock assessments. In Proceedings from a workshop on modeling protogynous hermaphroditic fishes; Raleigh, NC, 29-30 August 2012 (G. Shepherd, K. Shertzer, J. Coakley, and M. Caldwell, eds.), p. 5-9. Mid-Atlantic Fishery Management Council, Dover, DE. [Available at website, accessed May 2015.] changing sex to male as needed. The result is a sex ratio dominated by females at small sizes or young ages and males at large sizes or old ages (Warner, 1975; Allsop and West, 2004; Munday et al., 2006). It is typical in a protogynous species for the large, dominant male to monopolize spawning, although there is a wide range of behavioral roles for secondary males that may allow participation (Petersen, 1991). The resulting sex ratio, highly skewed toward males at large sizes, tends to make protogynous species vulnerable to size-selective fisheries, possibly resulting in a shortage of males-a shortage that limits the potential for egg fertilization (Huntsman and Schaaf, 1994; Heppell et al., 2006).

Characteristics of hermaphroditic species make them particularly challenging to assess and manage. Management of exploited fish stocks often is guided by the results of population dynamic models (Quinn and Deriso, 1999; Haddon, 2001; Brander, 2003), and basic models are predicated on certain assumptions about the life history of a species, including the ex- 
pectation that both sexes will be equally vulnerable to exploitation. Along with other possible scenarios, such as differential spatial patterns by sex or significant sexual dimorphism, hermaphroditic life histories often violate this assumption, and unique analytical approaches may be warranted. Implications of a protogynous life history for stock assessments and management have been explored previously (Alonzo and Mangel, 2004; Heppell et al., 2006; Brooks et al., 2007; Alonzo et al., 2008). The conclusion from simulation models, as well as empirical observations, is that populations subjected to sex-specific exploitation as a consequence of having a hermaphroditic life history are at a higher risk of overexploitation than are gonochoristic species (Alonzo and Mangel, 2004; Heppell et al., 2006; Hamilton et al., 2007). Thus developing management measures for a protogynous species on the basis of the assumptions implicit for gonochoristic species could possibly result in overfishing.

The black sea bass (Centropristis striata) is a protogynous hermaphroditic species that is distributed from the Gulf of Maine to the Gulf of Mexico (Lavenda, 1949; Collette and Klein-MacPhee, 2002) and is managed as 3 unique stocks segregated by Cape Hatteras, North Carolina, and the Florida Keys (Bowen and Avise, 1990; Roy et al., 2012; McCartney et al., 2013). Each stock is subjected to commercial and recreational exploitation and is primarily caught in trawl gear and fish pots, as well as on hook-and-line gear in the coastal regions (Shepherd and Terceiro, 1994). Black sea bass in the South Atlantic and Gulf of Mexico are not migratory and are commonly associated with local structured habitat (Drohan et al., 2007). In contrast, black sea bass in the Middle Atlantic (i.e., the northern stock) undergo seasonal migrations of up to 300 $\mathrm{km}$, moving from the continental shelf or slope edge in winter to the coastal waters in spring and summer (Musick and Mercer, 1977; Moser and Shepherd, 2009).

Life history characteristics vary between the 2 southern stocks and the northern stock. Spawning in the Middle Atlantic stock occurs within a relatively limited period compared with the spawning period of black sea bass in the southern stocks. In all 3 stocks, dominant males in spawning aggregations are characterized by a prominent nuccal hump and bright blue coloration around the hump and eyes (Lavenda, 1949); however, in the northern stock, additional males in ripe gonadal condition but lacking the secondary sex characteristics are also present in the spawning areas (NEFSC ${ }^{2}$. These features are typical of secondary males that may attempt to fertilize the eggs of females spawning with dominant males (Pitcher, 1993; Taborsky, 1994; Young et al., 2013). Empirical data collected during bottom trawl surveys of the Northeast Fisheries Science Center (NEFSC), National Marine Fisheries Service, in-

\footnotetext{
2 NEFSC (Northeast Fisheries Science Center). 2012. $53^{\text {rd }}$ Northeast regional stock assessment workshop (53 rd SAW) assessment report. Northeast Fish. Sci. Cent. Ref. Doc. 1205, 559 p. [Available at website, accessed May 2015.]
}

dicate that about $30 \%$ of black sea bass at each size below $30 \mathrm{~cm}$ (all lengths in this manuscript refer to total length) were male and that a significant proportion (i.e., up to $45 \%$ ) of black sea bass at sizes above $45 \mathrm{~cm}$ were female $\left(\mathrm{NEFSC}^{2}\right)$. Additionally, growth in this stock appears to be similar for males and females. The characteristics of the black sea bass of the northern stock, such as a prematurational sex change, the presence of mature secondary males, and the relative abundance of large females $(>45 \mathrm{~cm}$ ), distinguish them from typical protogynous hermaphrodites.

The northern Atlantic population of black sea bass has been evaluated in stock assessments to support fishery management programs $\left(\mathrm{NEFSC}^{2}\right)$. One of the main uncertainties cited in relation to the development of management measures is the implication of a protogynous life history. Specifically, there is concern that the removal of large males $(>45 \mathrm{~cm}$ ) would significantly affect the reproductive capability of the stock, thereby making it particularly vulnerable to exploitation. Implicit in that concern is that only the large males reproduce or, if not, the large females change sex to compensate for the reduced availability of large males.

Although methods for stock assessment of protogynous populations have been evaluated, in several studies, the implications of the possible contribution of secondary males to spawning in a population have not been explored fully. In particular, the response to exploitation of a stock with functional secondary males that result from a prematurational sex change has not been tested to determine whether the stock is more or less vulnerable to overexploitation than a gonochoristic or typical protogynous stock with similar population attributes. Our objective was to develop a population model based on the northern stock of black sea bass to evaluate the robustness of an atypical protogynous population in response to fishery exploitation. We do not intend to provide new methods for stock assessments of hermaphroditic species but rather to improve the understanding of the relationship between the life history of a species and its ability to withstand exploitation.

We developed a length-based population model to evaluate the impact of a range of exploitation intensities on population persistence and catch, under different theoretical life history scenarios. We considered an experimental atypical protogynous (AP) population with 3 scenarios: 1) no mature secondary males contribute to spawning (AP-0); 2) 50\% of mature secondary males participate in spawning (AP-50); and 3) $100 \%$ of mature secondary males participate in spawning (AP100). Note that our use of the term secondary simply implies that these males are not visibly dominant (i.e., secondary males lack the prominent nuccal hump and bright blue coloration). We did not make any assumptions about the behavior of secondary males except that some of them are able to spawn.

Results from simulations for the AP population were compared against those for equivalent gonochoristic (G) and typical protogynous (TP) populations, 
where spawning is monopolized by the dominant males. The model was structured to reflect black sea bass life history, and parameters were derived from empirical data when possible. Sex ratio characteristics for the AP population were based on the northern stock of black sea bass, and the TP population follows a biased sex ratio expected among protogynous species (Provost and Jensen ${ }^{1}$ ) with recruits being $100 \%$ female and all fish transitioning to male by the time they reach the maximum size in the model. We examined stock performance in the form of relative stock size, catch, spawning stock biomass (SSB), and number of recruits $(R)$ under increasing levels of fishing-induced mortality [hereafter: fishing mortality] $(F)$, and we ran sensitivity analyses to evaluate the effect of different capture probabilities, female-to-male transition rates, proportions of secondary versus dominant males, and recruitment scenarios, as well as the effect of possible spawning by secondary males in the TP population.

\section{Materials and methods}

\section{Model structure}

A length-structured model was developed to compute the abundance at length of fish in 4 life stages $(i)$ over time: females $(i=U)$, transitionals $(i=T)$, secondary males $(i=S)$, and dominant males $(i=D)$. The model follows a monthly time step where each time period $(t)$ is equivalent to the corresponding year $(y)$ and month $(m)$ (i.e., $t=$ month $m$ in year $y$ ).

Recruits $(\leq 11 \mathrm{~cm})$ in the model are initially all female but some immediately undergo a change into male, according to populationspecific recruit sex ratios. Female and male recruits can follow several potential trajectories over their lifetime (Fig. 1). Female recruits $(R U)$ will either remain female over their entire life or become transitionalphase fish [transitionals] for a single time step followed by transformation into either secondary or dominant males. Male recruits $(R S)$ enter the population as secondary males and either remain secondary males or become dominant males (without a transition phase). Therefore, at any time $t$, the abundance at length $l$ of fish at a specific life stage is the sum of recruits of the given sex, of individuals entering the stage, and of individuals that were previously in that stage and survived:

$$
\begin{gathered}
U_{\mathrm{lt}}=R U_{\mathrm{lt}}+U_{\mathrm{Ult}}, \\
T_{\mathrm{lt}}=T_{\mathrm{Ult}}, \\
S_{\mathrm{lt}}=R S_{\mathrm{lt}}+S_{\mathrm{Tlt}}+S_{\mathrm{Slt}},
\end{gathered}
$$

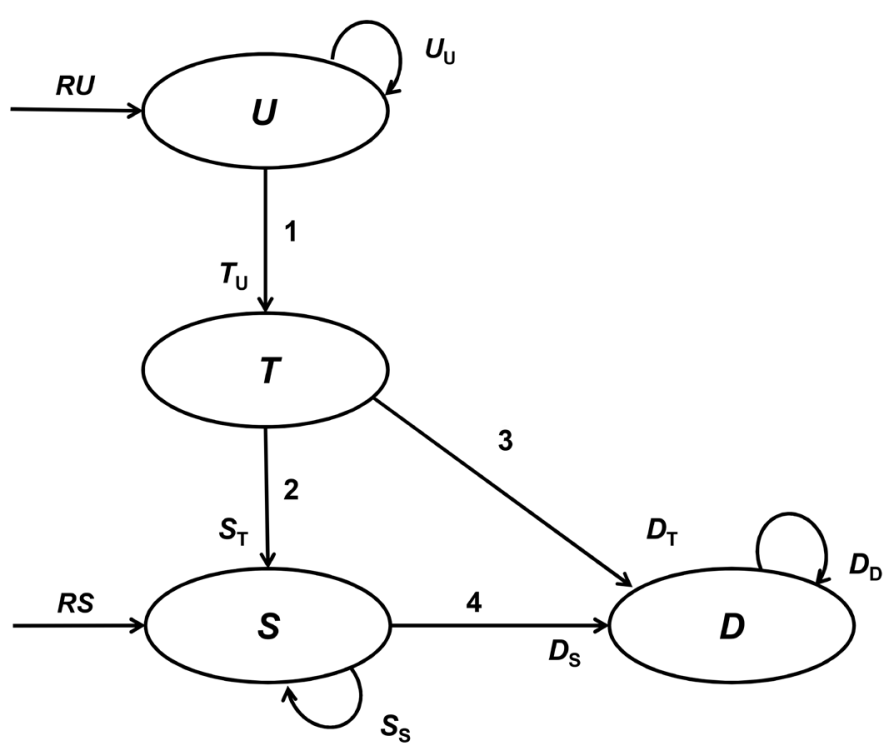

Figure 1

Diagram of possible trajectories through 4 different life stages of black sea bass (Centropristis striata) (female $[U]$, transitional $[T]$, secondary male $[S]$, and dominant male $[D]$ ) in the lengthbased model developed to study the response to exploitation by gonochoristic, atypical protogynous, and typical protogynous populations. Recruits are either female $(R U)$ or male $(R S)$, and there are 4 types of transitions in which a fish can transform into a different life stage by the next time period: 1) a female can become a transitional, 2) a transitional can become a secondary male, 3) a transitional can become a dominant male, or 4) a secondary male can become a dominant male. Subscripts letters $U, T, S$, and $D$ indicate the life stage at the previous time step. When available, parameter and other values used in the model came from data collected during bottom trawl surveys conducted by the NOAA Northeast Fisheries Science Center during 1984-2013.

$$
D_{\mathrm{lt}}=D_{\mathrm{Tlt}}+D_{\mathrm{Slt}}+D_{\mathrm{Dlt}},
$$

where the subscripts $U, T, S$, and $D$ indicate life stage at the previous time step (for example, $S_{\mathrm{T}}$ refers to the portion of all secondary males at time $t$ that were transitionals in the previous month). Total population abundance over all lengths, $N_{\mathrm{t}}$, is then calculated as

$$
N_{\mathrm{t}}=\sum_{l=1}^{l_{\max }}\left(U_{\mathrm{lt}}+T_{\mathrm{lt}}+S_{\mathrm{lt}}+D_{\mathrm{lt}}\right),
$$

where $l_{\max }=$ the maximum length of a fish in the population.

From one time step to the next, processes affecting the population occur in the following order: mortality (including natural mortality $[M]$ and $F$ ), transition, and growth. At each time step, SSB (calculated by using maturity-at-length and length-weight equations), recruitment, and catch are determined.

Natural mortality at time $t\left(M_{\mathrm{t}}\right)$ is assumed constant across stages, lengths, and months; therefore, 


$$
M_{\mathrm{t}}=\frac{M_{\mathrm{y}}}{12},
$$

where $M_{\mathrm{y}}=$ total annual natural mortality for year $y$.

Fishing mortality for length $l$ and time $t$ is defined as

$$
F_{1 \mathrm{t}}=\frac{F_{\mathrm{y}}}{12} \delta_{\mathrm{l}},
$$

where $F_{\mathrm{y}}=$ total annual fishing mortality for year $y$; and

$\delta_{1}=$ the probability of capture (i.e., capture selectivity) at length $l$ defined by a logistic 2-parameter model,

$$
\delta_{1}=\left(1+e^{\left(-\frac{1-\alpha}{\beta}\right)}\right)^{-1},
$$

where $\alpha=$ the length of the fish with $50 \%$ probability of being retained $\left(L_{50}\right)$; and

$\beta=$ the slope of the selectivity curve $\delta_{1}$.

It follows that catch in numbers for stage $i$ at length $l$ at time $t$ is

$$
C_{\mathrm{ilt}}=\left(\frac{F_{\mathrm{lt}}}{F_{\mathrm{lt}}+M_{\mathrm{t}}}\right) N_{\mathrm{ilt}}\left(1-e^{-\left(F_{\mathrm{lt}}+M_{\mathrm{t}}\right)}\right) .
$$

In the protogynous population scenarios, we assumed transitions (Fig. 1) occur only after spawning in month 11 or 12 , where a fish can transform from a female to a transitional $(m=11)$, from a transitional to a secondary male $(m=12)$, from a transitional directly to a dominant male $(m=12)$, or from a secondary male to a dominant male $(m=12)$. For simplicity, fish can be in the transitional stage only during one time step. The probability of an individual of stage $i_{1}$ and length $l$ in month $m$ becoming stage $i_{2}$ in the following month is represented by $\theta_{1 \mathrm{~m}}^{\mathrm{i}_{1} i_{2}}$. Transition rates are all zero for the $\mathrm{G}$ population because no sex change occurs; the sex ratio for this scenario is fixed at 1:1.

Growth is assumed constant across stages and sexes but varies annually by quarter, $(q)$. Growth occurs at the start of each quarter and is defined by a lower triangular matrix $\left(G_{q}\right)$ with dimensions $l_{\max } \times l_{\max }$, in which each element $g_{\mathrm{L} 2, \mathrm{~L} 1, \mathrm{q}}$ represents the probability that a fish of length $L 1$ at the start of quarter $q$ will grow to length $L 2$ by the start of the following quarter. Therefore, the abundance of individuals of stage $i_{2}$ at length $L 2$ at time $t, N_{\mathrm{i}_{2} \mathrm{~L} 2 \mathrm{t}}$, is calculated as the number of individuals of stage $i_{1}$ at length $l$ (where $l \leq L 2$ ) at time $(t-1)$ that survived, that transitioned to stage $i_{2}$, and that grew to length $L 2$, summed over all values of $l$ :

$$
N_{\mathrm{i}_{2} \mathrm{lt}}=\sum_{l=1}^{L 2}\left[N_{\mathrm{i}_{1}(\mathrm{t}-1)} e^{\left(-\left(\mathrm{M}_{\mathrm{t}}+F_{\mathrm{lt}}\right)\right)} \theta_{\mathrm{lm}}^{\mathrm{i}_{\mathrm{i}} \mathrm{i}_{2}} g_{\mathrm{L} 2, \mathrm{l}, \mathrm{m}}\right] .
$$

Given the uncertainty resulting from defining SSB as female only (Brooks et al., 2007), recruitment levels are determined from male and female spawning stock as of September $(m=9)$ and from recruits that entered the population in October $(m=10)$. Maturity is specified by using sex-specific maturity ogives defined by a 4-parameter logistic equation, as follows:

$$
O_{\mathrm{j} 1}=\frac{a_{\mathrm{j}}-d_{\mathrm{j}}}{1+\left(\frac{l}{c_{\mathrm{j}}}\right)^{b_{\mathrm{j}}}}+d_{\mathrm{j}},
$$

where $O_{\mathrm{j} 1}=$ the proportion of fish of $\operatorname{sex} j$ (where $j=U$ for females, $j=V$ for males) and length $l$ that are mature;

$a_{\mathrm{j}}=$ the minimum asymptote parameter for sex $j$

$b_{\mathrm{j}}=$ the slope parameter for sex $j$;

$c_{\mathrm{j}}=$ the inflection point (i.e., length at $50 \%$ maturity) parameter for sex $j$; and

$d_{\mathrm{j}}=$ the maximum asymptote parameter for $\operatorname{sex} j$.

Spawning stock biomass is the sum of female and male spawning biomass (i.e., biomass of mature fish) for the $\mathrm{G}$ population and is the sum of female and dominant male spawning biomass for the TP scenario. For the AP population scenarios, SSB is the sum of female and dominant male spawning biomass, plus the spawning biomass of the given proportion $(0 \%, 50 \%$, or $100 \%)$ of secondary males.

The lack of an accepted stock-recruitment model for black sea bass, combined with the desire to include the SSB sex ratio as a factor influencing recruitment, led to the development of an approach that involves recruitment bins. With this approach, depending on the value of SSB and the sex ratio (in numbers) within the spawning stock, recruitment abundance for year $y\left(R_{\mathrm{y}}\right)$ was randomly drawn (on the basis of a random number seed) from 1 of 3 cumulative distribution functions (CDF) representing 3 productivity bins. Each CDF was created from a lognormal distribution generated by using a mean recruitment and standard deviation from empirical data.

As long as the proportion of females (PropF) and males (Prop $M)$ in the spawning stock remained above a sex ratio threshold $\left(H_{1}\right)$ (i.e., as long as the sex ratio in the spawning stock was not overly skewed), recruitment was drawn according to the value of SSB in relation to $3 \mathrm{SSB}$ breakpoints, $B_{\min }, B_{1}$, and $B_{2}$, which delimit the productivity bins. Recruitment values associated with $B_{\min } \leq \mathrm{SSB}<B_{1}$ represent the low productivity bin, recruitment values associated with $B_{1} \leq$ $\mathrm{SSB}<B_{2}$ represent the medium productivity bin, and recruitment values associated with $\mathrm{SSB} \geq B_{2}$ represent the high productivity bin. If PropF or PropM dropped below $H_{1}$ but remained above a minimum sex ratio threshold $\left(H_{\min }\right)$, recruitment was drawn from the low productivity bin. There was no recruitment (i.e., $R_{\mathrm{y}}=0$ ) when SSB $<B_{\min }$ or if either PropF or Prop $M$ dropped below $H_{\text {min }}$. As mentioned above, recruits can enter the population either as females or secondary males. Respectively, if $m=10$, the abundance of female and secondary male recruits at length $l$ at time $t, R U_{\mathrm{lt}}$ and $R S_{\text {lt }}$, are calculated as 


\section{Table 1}

Values and descriptions of parameters used in the length-based population model developed to study the response to exploitation by gonochoristic, atypical protogynous, and typical protogynous populations of black sea bass (Centropristis striata).

\begin{tabular}{|c|c|c|}
\hline Parameter & Value & Description \\
\hline$R_{1}$ & $15,000,000$ & Initial number of recruits (i.e., initial population) \\
\hline$M_{\mathrm{y}}(y=1-5)$ & 0.01 & Annual full natural mortality rate for year $y$, initial years \\
\hline$M_{\mathrm{y}}(y=6-45)$ & 0.40 & Annual full natural mortality rate for year $y$ \\
\hline$F_{\mathrm{y}}(y=1-14)$ & 0.00 & Annual fishing mortality rate for year $y$, initial years \\
\hline$F_{\mathrm{y}}(y>14)$ & $\{0.00,0.10, \ldots 1.50\}$ & Annual fishing mortality rate for year $y$ \\
\hline$\alpha$ & 27.09 & Length $(\mathrm{cm})$ of $50 \%$ probability of retention by the gear $\left(L_{50}\right)$ \\
\hline$\beta$ & 1.053 & Slope of fishing selectivity curve $\delta_{l}$ \\
\hline$B_{\min }$ & 690 & Minimum spawning stock biomass (SSB; metric tons $[\mathrm{t}]$ ) required for potential recruitment \\
\hline$B_{1}$ & 2,955 & SSB Breakpoint $1(\mathrm{t})$ \\
\hline$B_{2}$ & 7,388 & SSB Breakpoint $2(\mathrm{t})$ \\
\hline$H_{\min }$ & 0.02 & Minimum sex ratio threshold (proportion female or male in spawning stock) \\
\hline$H_{1}$ & 0.10 & Sex ratio threshold 1 (proportion female or male in spawning stock) \\
\hline$a_{\mathrm{U}}$ & 0.000 & Minimum asymptote parameter of female maturity ogive $O_{\mathrm{Ul}}$ \\
\hline$b_{\mathrm{U}}$ & 6.569 & Slope (steepness) parameter of female maturity ogive $O_{\mathrm{Ul}}$ \\
\hline$c_{\mathrm{U}}$ & 21.233 & Inflection point parameter of female maturity ogive $O_{\mathrm{Ul}}$ \\
\hline$d_{\mathrm{U}}$ & 1.000 & Maximum asymptote parameter of female maturity ogive $O_{\mathrm{Ul}}$ \\
\hline$a_{\mathrm{V}}$ & 0.000 & Minimum asymptote parameter of male maturity ogive $O_{\mathrm{Vl}}$ \\
\hline$b_{\mathrm{V}}$ & 6.374 & Slope (steepness) parameter of male maturity ogive $O_{\mathrm{Vl}}$ \\
\hline$c_{\mathrm{V}}$ & 18.506 & Inflection point parameter of male maturity ogive $O_{\mathrm{Vl}}$ \\
\hline$d_{\mathrm{V}}$ & 1.000 & Maximum asymptote parameter of male maturity ogive $O_{\mathrm{Vl}}$ \\
\hline$L_{\infty}$ & 56.7 & Asymptotic maximum length $(\mathrm{cm})$ \\
\hline$K$ & 0.0585 & Growth coefficient (per seasonal quarter) \\
\hline$q_{0}$ & 1.888 & Theoretical age (in seasonal quarter units) at length $l=0 \mathrm{~cm}$ \\
\hline$C$ & 0.026 & Oscillation amplitude coefficient for seasonal growth model \\
\hline$s_{1}$ & -0.2794 & Sine wave starting time coefficient (in seasonal quarter units) for the seasonal growth model \\
\hline$v_{1 \mathrm{U}}=v_{1 \mathrm{~T}}$ & -10.912 & Weight-at-length coefficient for female $(U)$ and transitional $(T)$ stages \\
\hline$v_{2 \mathrm{U}}=v_{2 \mathrm{~T}}$ & 2.9120 & Weight-at-length exponent for female $(U)$ and transitional $(T)$ stages \\
\hline$v_{1 \mathrm{~S}}=v_{1 \mathrm{D}}$ & -10.954 & Weight-at-length coefficient for secondary $(S)$ and dominant $(D)$ male stages \\
\hline$v_{2 \mathrm{~S}}=v_{2 \mathrm{D}}$ & 2.9094 & Weight-at-length exponent for secondary $(S)$ and dominant $(D)$ male stages \\
\hline
\end{tabular}

$$
\begin{gathered}
R U_{\mathrm{lt}}=R_{\mathrm{y}} p_{\mathrm{l}}\left(1-\rho_{\mathrm{l}}\right), \\
R S_{\mathrm{lt}}=R_{\mathrm{y}} p_{\mathrm{l}} \rho_{\mathrm{l}},
\end{gathered}
$$

where $p_{1}=$ the proportion of recruits at length $l$; and $\rho_{1}=$ the proportion of male recruits at length $l$.

If $m \neq 10$, then $R U_{\mathrm{lt}}=R S_{\mathrm{lt}}=0$.

Finally, weight at length for a fish of stage $i$ and length $l\left(W_{i 1}\right)$ was modeled with the allometric relationship:

$$
W_{\text {il }}=\exp \left(\ln \left(v_{1 \mathrm{i}}\right)+v_{2 \mathrm{i}} \ln (l)\right)
$$

where $v_{1 \mathrm{i}}=$ the weight-at-length coefficient for stage $i$;

$v_{2 \mathrm{i}}=$ the weight-at-length exponent for stage $i$; and

$v_{1 \mathrm{i}}$ and $v_{2 \mathrm{i}}$ are constants under the assumption of isometric growth.

\section{Parameter estimation and input values for models}

All parameter and input values were estimated on the basis of empirical data from the northern stock of black sea bass (an exploited stock) where appropriate.
Estimated model parameters specifying $M$, selectivity, maturity, and weight at length were identical for all experimental populations, as were growth, initial population size, and recruitment inputs (Table 1). Transition rates and size at transition were specific to each experimental population, resulting in differences in the proportion of male recruits and population sex ratio.

For the first 5 years, $M_{\mathrm{y}}$ was set to 0.01 to initialize the simulated populations and for subsequent years was held constant and equal to 0.4 (Shepherd $^{3}$ ). Initially, $F_{\text {y }}$ was 0.00 , after which $F_{y}$ was set to different levels as described later in the Simulations and sensitivity analyses section. Selectivity parameters were estimated from assessment results (Shepherd ${ }^{3}$ ). Maturity ogives for females and males were defined on the basis of maturity data from the NEFSC spring survey cruise (1984-2013; $n=3285)$, and weight-at-length parameters were estimated by using results from the NEFSC bottom trawl surveys conducted in spring and fall, 19932013 (Shepherd $^{3}$ ).

\footnotetext{
${ }^{3}$ Shepherd, G. R. 2009. Black sea bass 2009 stock assessment update. Northeast Fish. Sci. Cent. Ref. Doc. 09-16, 30 p. [Available at website, accessed May 2015.]
} 
Quarterly growth matrices were derived from average length-at-age data from the NEFSC spring (19842011, $n=3026)$, fall $(1983-2010, n=3381)$, and winter (1992-2007, $n=3390)$ surveys, and from commercial catch data from a summer survey $(2012, n=59)$. Quarterly average length at age from all seasonal quarters were initially fitted (nonlinear regression, SAS 9.44, SAS Institute Inc., Cary, NC) to a von Bertalanffy growth curve for length in quarter $q$,

$$
L_{\mathrm{q}}=L_{\infty}\left(1-e^{-\mathrm{K}\left(\mathrm{q}-\mathrm{q}_{0}\right)}\right) \text {. }
$$

Data were then fitted to a seasonal growth model by using parameter values from the von Bertalanffy growth model as defined in Pitcher and MacDonald (1973):

$$
L_{\mathrm{q}}=L_{\infty}\left(1-e^{-\mathrm{K}_{1}}\right) \text {, }
$$

where

$$
K_{1}=C \sin \left(\frac{2 \pi\left(q-s_{1}\right)}{52}\right)+K\left(q-q_{0}\right),
$$

where

$$
\begin{aligned}
C= & \text { the amplitude of the oscillation } \\
& \text { around the average (nonseasonal) } \\
& \text { growth curve; and } \\
s_{1}= & \text { the starting time for the sine wave. }
\end{aligned}
$$

Expected quarterly growth by length was calculated for each centimeter from the sine wave growth model and formed the basis for deriving the quarterly growth matrices, which also were calibrated to empirical length distributions by quarter. Growth was fastest during the summer (quarter 3) and almost negligible during winter (quarter 1) -findings that are consistent with those from another study (Able and Hales, 1997).

Initial recruitment in year 1 was 15 million individuals, a value scaled to produce catch at equilibrium equivalent to empirical estimates at corresponding $F$ values $\left(\right.$ Shepherd $\left.{ }^{3}\right)$. The length distribution of recruits was based on the average length distribution (at age zero), from NEFSC fall surveys, for lengths 4-11 cm for 2009-2013 $(n=214)$. The 3 recruitment productivity bins were structured to reflect the distribution of SSB and recruits in the most recent assessment of the northern stock of black sea bass (Shepherd $\left.{ }^{3}\right)$. We defined 3 overlapping lognormal distributions (1 for each bin) and randomly drew 500 values from each distribution to create a CDF for each productivity bin. The average (and standard deviation [SD]) of the 500 $R$ values per bin were 6517.9 individuals (SD 3058.0) for the low recruitment bin, 13,912.1 individuals (SD 4057.0) for the medium recruitment bin, and 29,634.3 individuals (SD 9289.1) for the high recruitment bin. Sex ratio thresholds $\left(H_{\min }\right.$ and $\left.H_{1}\right)$ were set to 0.02 and 0.10 , respectively; these values were assumed reasonable because no empirical data were available.

Input data specifying the proportion of male recruits and the transitions between the 4 different stages (i.e., female-transitional-secondary-dominant) differed among the 3 experimental populations. For the G case,

\footnotetext{
${ }^{4}$ Mention of trade names or commercial companies is for identification purposes only and does not imply endorsement by the National Marine Fisheries Service, NOAA.
}

half of the recruits were males $\left(\rho_{1}=0.5\right.$; Fig. $\left.2 \mathrm{~A}\right)$ for a population with only females and males (i.e., transition rate from female to transitional $\left[\theta^{\mathrm{UT}}{ }_{1 \mathrm{~m}}\right]$ and transition rate from secondary to dominant male $\left[\theta^{\mathrm{SD}}{ }_{\mathrm{lm}}\right]$ were equal to zero). For the protogynous populations, the sex ratio of recruits and transition rates were determined such that the sex ratios of the AP population would reflect empirical data for the northern stock of black sea bass from the NEFSC bottom trawl surveys $(n=13,107)$ conducted in spring $(1984-2013, n=4040)$, fall (1983-2013, $n=5126)$, and winter (1992-2007, $n=3941$ ), and the sex ratio of the TP population would have a characteristic pattern with all female recruits and all fish transitioning to male by $65 \mathrm{~cm}$ (Fig. 2C).

Transition rates were identified with $F$ set at 0.35 to approximate the $F$ that was likely occurring when the empirical data were collected. In the AP population, $23 \%$ of recruits were male and $\theta^{\mathrm{UT}} 1 \mathrm{~m}$ were equal to zero for lengths below $15 \mathrm{~cm}$ and followed a linear relationship (with slope 0.0039 and intercept -0.0492) for lengths 15 to $65 \mathrm{~cm}$. The TP population had zero male recruits, and $\theta^{\mathrm{UT}} \mathrm{lm}$ were zero for lengths below 12 $\mathrm{cm}$, approximated a linear trend with slope 0.0108 and intercept -0.0228 for lengths $12-43 \mathrm{~cm}$, and were constant at 0.43 for lengths $44-65 \mathrm{~cm}$. Because of a lack of data to inform the split into secondary and dominant males in the protogynous populations, we assumed that half of males were dominant at $60 \mathrm{~cm}$ and set transition rates accordingly. Transition rates from transitional to dominant male $\left(\theta^{\mathrm{TD}}\right.$ lm $)$ and from secondary to dominant male $\left(\theta^{\mathrm{SD}}{ }_{1 \mathrm{~m}}\right)$ were held constant for all protogynous populations according to a linear relationship that was derived from the data, with slope 0.0109 and intercept -0.1522 for lengths 15 to $60 \mathrm{~cm}$. A constant transition rate of 0.5 was applied for lengths greater than $60 \mathrm{~cm}$.

\section{Simulations and sensitivity analyses}

For each experimental population, the model was run for 45 years with values of annual $F$ ranging from 0.0 to 1.5 by increments of 0.1 (i.e., 16 constant $F$ scenarios). Each model configuration was run 100 times, with random draws of recruitment values $\left(R_{\mathrm{y}}\right)$. Results from year 45 were assumed to be representative of the populations at equilibrium under each configuration. For each scenario, the average and $95 \%$ confidence interval (CI) for stock abundance, stock biomass, catch abundance, catch biomass, $\mathrm{SSB}$, and $R$ were calculated at each value of $F$. Stock results represent abundance on 1 May $(m=5)$, and catch results represent the sum of catch over the entire year.

Additional runs were performed to evaluate the sensitivity of the model results to capture selectivity, rates of sex change by females, proportion of dominant males, sex ratio thresholds of the spawning stock, and inclusion of the TP secondary males in SSB. For selectivity, the AP-50 configuration was run with values of $\pm 10 \%(24.38 \mathrm{~cm}$ and $29.8 \mathrm{~cm})$ for parameter $\alpha\left(L_{50}\right)$ of the selectivity curve $\delta_{1}$ (Eq. 8). To assess the influence 

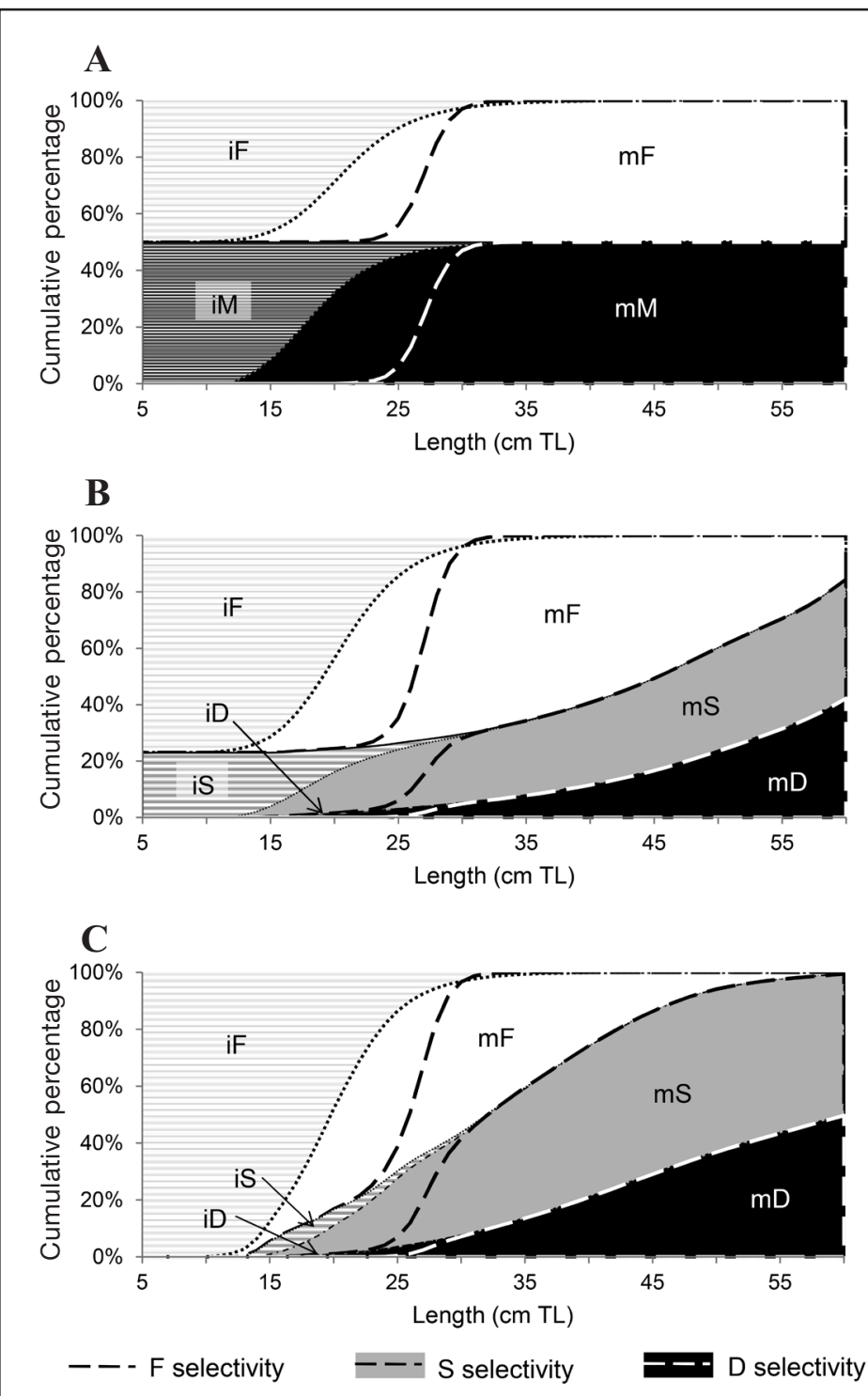

Figure 2

Cumulative percentages of life stages of black sea bass (Centropristis striata) by length (centimeters in total length [TL]) for (A) gonochoristic, (B) atypical protogynous, and (C) typical protogynous populations, which were simulated to study response to exploitation by using a length-based population model. Each shade of gray represents a different maturity state, immature (i) and mature $(\mathrm{m})$, and life stage, including female (F), secondary male (S), dominant male (D), or simply male (M), in the gonochoristic case. Dashed lines represent fishing selectivity curves for each stage, as indicated in the key. Life stage characteristics surveys conducted by the NOAA Northeast Fisheries Science Center during 1984-2013. were based on data collected, when available, from bottom trawl

rates that resulted in $25 \%$ and $75 \%$ males at $60 \mathrm{~cm}$ being dominant, respectively. Another set of sensitivity runs evaluated the effect of adding a third sex ratio threshold for the spawning stock $\left(H_{2}\right)$. This additional threshold acts as a penalty for moderately skewed sex ratios in the spawning stock by directing $R_{\mathrm{y}}$ to be drawn from the medium productivity bin if PropF or PropM is between $H_{1}$ (where $H_{1}=0.20$ ) and $\mathrm{H}_{2}$; all population simulations were rerun with $H_{2}=0.40$. Finally, the effect of spawning by secondary males in the TP population was explored with runs allowing participation in spawning by $50 \%$ and $100 \%$ of mature secondary males.

\section{Results}

\section{Simulations}

All simulation results showed a similar pattern at equilibrium (year 45) of decreasing average stock size (Fig. 3) with increasing $F$, and of catch increasing with $F$, reaching a maximum, and then decreasing to zero as harvesting pressure rose (Fig. 4). However, the specific performance of each experimental population differed. A general pattern existed where the results for $\mathrm{G}$ and $\mathrm{TP}$ populations bounded the results from the $3 \mathrm{AP}$ populations, the size of which declined in an order consistent with the percentage of contributions to spawning made by secondary males. The performance of the AP-0 population was closest to the TP scenario; the AP-100 population generally did not differ from the G case, and the AP-50 population fell in between.

The size of the TP population at equilibrium remained well below levels reached by the $\mathrm{G}$ case at the same $F$, with stock size (abundance and biomass) never exceeding $81 \%$ of the size of the $\mathrm{G}$ population and remaining below $50 \%$ of the size of the $\mathrm{G}$ population for $F \geq 0.2$, before approaching zero at $F=1.0$ (Fig. 3 ). The equilibrium stock size of the AP-0 population started at almost the same size as that of the G population (94\%) under no exploitation but dropped to levels similar to those of the TP population once $F$ increased, even dropping below the size of the TP population for $F=0.2$ and $F=0.3$. The AP-50 equilibrium stock size was equivalent to that of the $\mathrm{G}$ population for low values of $F$ but declined at a faster rate as $F$ increased (Fig. 3). In contrast, the AP-100 stock size was almost of female transition rates, the AP-50 and TP configurations were run with values of $\pm 10 \%$ of $\theta_{\mathrm{lm}}^{\mathrm{UT}}$. We ran 2 sets of simulations on the protogynous populations to consider sensitivity of these populations to the proportion of males that were dominant, using transition identical to that of the G population for all values of $F$.

The difference in response of the AP populations stemmed from the increase in participation of secondary males in spawning-an increase that guarded against abrupt stock size decline under exploitation. 
For example, at $F=0.3$, stock abundance for the AP-0 population was $31 \%$ of the abundance of the G population, the abundance of the AP-50 population was $76 \%$ of the abundance of the G population, and the AP-100 population was $98 \%$ of the abundance of the $\mathrm{G}$ population (Fig. 3A); the corresponding stock biomass results were $23 \%, 82 \%$, and $100 \%$, respectively (Fig. $3 \mathrm{~B})$. Stock abundance approached zero at $F=1.1$ for the AP-0 population and at $F=1.4$ for the AP-50 population, and stock abundance of the AP-100 population collapsed above $F=1.5$.

Contributions of secondary males to spawning altered the $F$ associated with maximum catch. Catch for the $G$ population was highest at $F=0.3$ before declining more than $65 \%$ by $F=0.7$ and approaching zero near $F=1.5$ (Fig. 4). Maximum yield in the TP configuration was reached at a lower $F(0.2)$ with catch number equal to $42 \%$ of the maximum catch number for the G population (Fig. 4A) and with catch biomass equal to $45 \%$ of catch biomass of the G population (Fig. 4B). Catch and $F$ at maximum catch in the AP scenarios increased with contributions of secondary males to spawning. The AP-0 population reached a maximum catch abundance of $34 \%$ of maximum $\mathrm{G}$ catch at $F=0.1$ (i.e., lower than the maximum of the TP population), and the AP-50 and AP-100 populations reached $81 \%$ and $99 \%$, respectively, of maximum $\mathrm{G}$ catch at $F=0.3$ (Fig. $4 \mathrm{~A}$ ); corresponding catch biomass amounts for the 3 populations were $41 \%, 83 \%$, and $100 \%$ (Fig. 4B).

Analysis of trends in SSB and $R$ (averages and 95\% CIs of the 100 simulation runs) versus $F$ provided information in relation to recruitment and indicated which productivity bin each population was drawing from at equilibrium (year 45) at different values of $F$ (Fig. 5). These results confirmed the pattern that, in general, results for the $\mathrm{G}$ and $\mathrm{TP}$ populations represented extremes in population productivity; the results of the 3 AP populations, however, occurred in a consistent order in between those extremes. Although all populations began in the high productivity recruitment bin $\left(\mathrm{SSB} \geq B_{2}\right)$ at $F=0.0$, the progression to the end of recruitment as $F$ increased and SSB fell below $B_{\text {min }}$ differed across populations. The $\mathrm{G}$ and AP-100 populations remained in the high productivity bin until $F=0.3$, at which point average recruitment declined to the medium and low productivity bins when $F=0.4-0.8$ and approached zero as $F$ reached 1.5 . The AP-50 population functioned un- der high productivity until $F$ reached 0.2 before following a similar decline and approaching recruitment failure at $F=1.2$. The TP and AP-0 scenarios stayed in the high productivity bin only if there was zero exploitation and SSB and $R$ declined more rapidly than they did for the other populations as $F$ increased, reaching recruitment failure by $F=0.9$.

As seen with the results of stock size discussed previously, the results for SSB and $R$ for the AP-0 population were below those of the TP population when $F=0.2$ and $F=0.3$. With increasing exploitation, females quickly constituted more than $90 \%$ of the spawning stock abundance for the AP-0 population (Fig. 2B), such that Prop $M$ fell between the sex ratio thresholds $\left(H_{\min }=0.02\right.$ and $H_{1}=0.10$ ), causing recruitment to drop to the low productivity bin (Fig 5B). In contrast, the TP popula- 
tion had a higher proportion of males in the spawning stock (Fig. 2C); therefore, the spawning stock sex ratio did not become overly skewed and did not trigger lower recruitment.

Stock-recruitment curves indicate that the TP and AP-0 populations had a lower productivity relationship than that of the G, AP-100, and AP-50 populations (Fig. 6). A lower line indicated that $R$ was drawn more often from the low recruitment bin because of an overly skewed spawning stock sex ratio (i.e., less than $10 \%$ males or less than $10 \%$ females).

\section{Sensitivity analyses}

The sensitivity analysis on capture selectivity for the AP-50 population produced the expected results. A $10 \%$ decrease in length at capture caused 1) lower stock size and earlier stock collapse (average decrease of $27 \%$ for stock abundance and $31 \%$ for stock biomass, with both reaching zero by $F=1.1$ ), 2) a higher (about $10 \%$ increase) maximum catch that occurred at a lower $F(0.2)$ but was followed by lower catch numbers, on average $36 \%$ (46\% for catch biomass) below the levels of the base AP-50 population, and 3) a general reduction in recruitment (46\% decrease in $R$ on average). On the other hand, a $10 \%$ increase in length at capture generated the opposite results: 1 ) stock size increased by about $53 \%$ (66\% for stock biomass) and did not collapse by $F=1.5 ; 2$ ) maximum catch was close to the results from the base AP-50 population and still occurred at $F=0.3$, and catch numbers for values of $F$ beyond where the maximum occurred were on average $55 \%$ (83\% for catch biomass) higher than the values for the base AP-50 population; and 3) $R$ increased by about $25 \%$.

Sensitivity to changes in $\theta_{\mathrm{lm}}^{\mathrm{UT}}$ differed for the AP50 and TP populations. All results indicate that stock performance for the AP-50 population had very low sensitivity to a $10 \%$ change in $\theta_{\mathrm{lm}}^{\mathrm{UT}}$, closely matching the results for the base AP-50 population. In contrast, the TP population was more sensitive to change in $\theta_{\mathrm{lm}}^{\mathrm{UT}}$. A $10 \%$ decrease in sex change transitions resulted in slightly higher stock sizes (7\% increase on average for stock number and stock biomass) and higher catch (9\% average increase in catch numbers and biomass), and a $10 \%$ increase in sex change transitions resulted in slightly lower stock sizes (8\% decrease on average for stock number and stock biomass) and lower catch $(9 \%$ average decrease in catch numbers and $8 \%$ average decrease for catch biomass); $F$ at maximum catch remained the same as it was for the base AP-50 population. Finally, drawing from recruitment bins was not affected by a $10 \%$ change in the sex transition rates.

Modifying the proportion of dominant versus secondary males in the protogynous populations had a greater effect on the AP-0 and TP populations than on the AP-50 configuration, and it had no effect on the AP-100 scenario because all males already contributed to spawning in this population. For the AP-50 population, reducing the proportion of dominant males to $25 \%$ at $60 \mathrm{~cm}$ led to an average decrease of just over $6 \%$ for stock abundance and catch at $F>0.2$ and to average drops of $8 \%$ in SSB and of $5 \%$ in $R$. With transition 

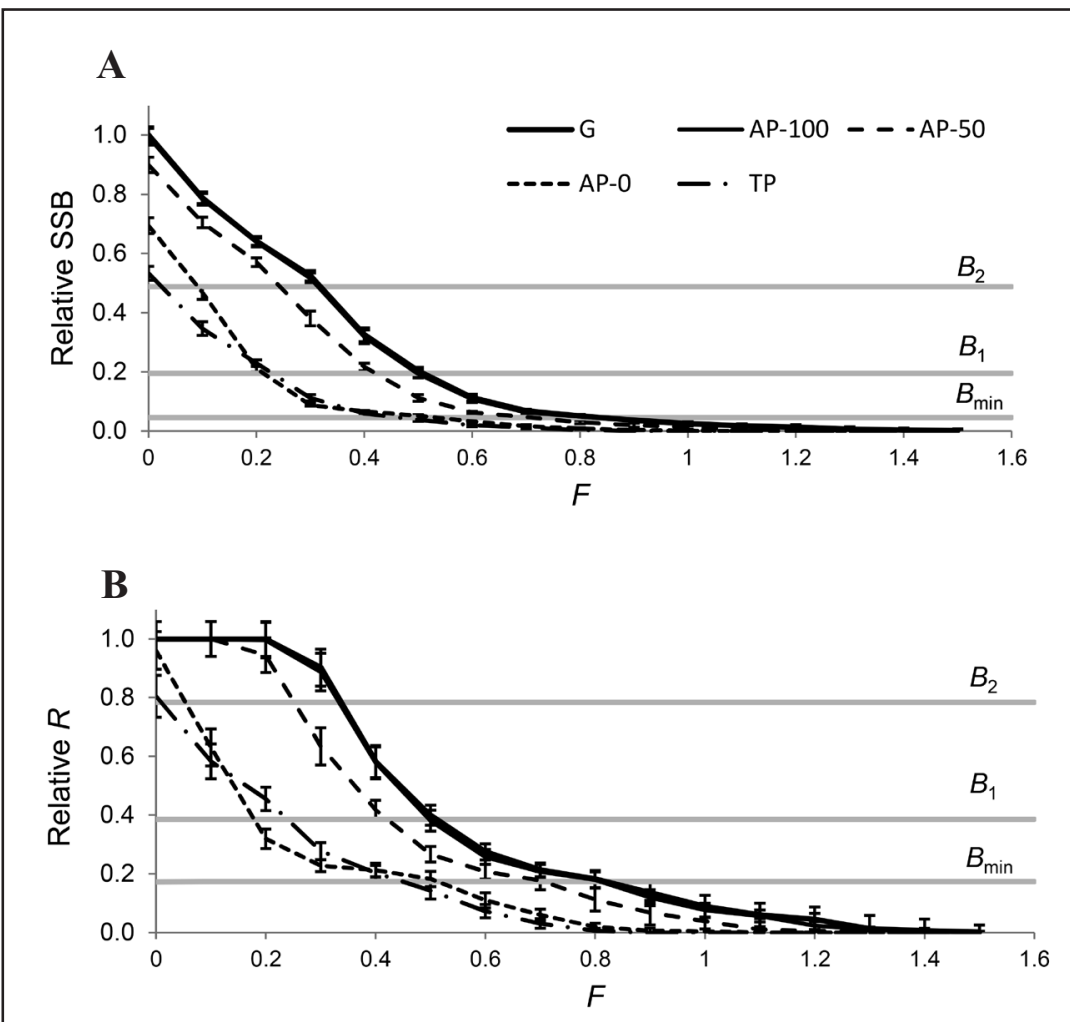

Figure 5

Equilibrium (year 45) (A) relative spawning stock biomass (SSB) and (B) relative number of recruits $(R)$ at values of fishing mortality $(F)$ from 0.0 to 1.5 by 0.1 increments for 5 experimental populations of black sea bass (Centropristis striata): a gonochoristic (G) population (thick, solid line), atypical protogynous (AP) populations with 100\% (AP-100; thin, solid line), 50\% (AP-50; dashed line), and 0\% (AP-0; dotted line) of mature secondary males contributing to spawning, and a typical protogynous population (TP; dashed-dotted line). Each line indicates the average SSB or $R$ (from 100 runs performed at each value of $F$ ) in relation to the average SSB or $R$ of the $\mathrm{G}$ population at $F=0.0$; vertical bars represent $95 \%$ confidence intervals. The gray reference lines indicate the position of the SSB breakpoints $\left(B_{\min }, B_{1}\right.$, and $\left.B_{2}\right)$ and corresponding $R$ delimiting the recruitment productivity bins. These results were obtained by using a length-based population model developed to study the response of these populations to exploitation.

configuration showed an average $62 \%$ decrease in SSB and $55 \%$ decrease in $R$. The increase in proportion of dominant males for the AP-0 population resulted in an increase in stock size and catch up to $70 \%$ at $F$ values below 0.3 and in an increase of around $45 \%$ for $F \geq 0$.3. The increase for the TP population was just over $30 \%$ for $F<0.3$ and over $50 \%$ for $F \geq 0.3$. On average, SSB increased by $60 \%$ and $R$ by $55 \%$ for both the AP-0 and TP populations. In general, the performance of the AP-0 and TP populations moved closer to that of the base $\mathrm{G}$ population with additional dominant males.

Sensitivity analyses that considered the effect of an alternative recruitment process indicated that most protogynous scenarios were sensitive to the addition of a third sex ratio threshold for the spawning stock, $\mathrm{H}_{2}$, where recruitment was drawn from the medium productivity bin if PropF or Prop $M$ was between $H_{1}=0.10$ and $H_{2}=0.40$. Because sex ratio in the $G$ population was fixed at $1: 1$, this population was not affected by any of the sex ratio thresholds. However, all the protogynous populations experienced a significant drop in recruitment with the addition of $H_{2}$, especially at lower $F$ values $(F \leq 0.7)$, where $R$ decreased by an average of $32 \%$ for the AP- 100 population, $28 \%$ for the AP-50 population, and $16 \%$ for the AP-0 and TP populations. At higher values of $F$, recruitment declined as well, but it did so less dramatically (from $0 \%$ to $19 \%$ ) because recruitment was already low as a result of low SSB at those levels of exploitation. Recruitment for the protogynous scenarios was limited to the low and medium productivity bins, indicating that the spawning stock sex

rates set for $75 \%$ of males being dominant at $60 \mathrm{~cm}$, the population experienced an average increase of $4 \%$ in stock abundance and catch and a rise in SSB and $R$ of about $5 \%$.

In contrast, the 2 populations with no secondary males contributing to spawning were more sensitive to a change in proportion of dominant males. With transition rates set for $25 \%$ of males being dominant at $60 \mathrm{~cm}$, stock size and catch for the AP-0 population dropped by around $65 \%$ for $F$ values below 0.3 and by just above $50 \%$ for $F \geq 0.3$. The TP population stock size and catch dropped by about $40 \%$ for $F$ values below 0.3 and by close to $65 \%$ for $F \geq 0.3$. Average SSB for the AP-0 population decreased by $58 \%$, and $R$ declined by 52\%; corresponding values for the TP ratio of these populations was always skewed (Fig. 2), specifically that Prop $M$ was always less than $H_{2}=0.4$.

In parallel to the decrease in recruitment, all other measures of stock performance (SSB, stock size, and catch) decreased as well. In general, all results for the AP populations moved closer to the results for the TP population, while continuing to follow the order that the TP population was least productive, followed by the AP-0, AP-50, and AP-100 populations, as seen in the results from the runs of the base model.

In the final sensitivity analysis, the effect of spawning by secondary males in the TP population was considered. Allowing all secondary males to spawn brought the TP population performance close to the performance of the base G and AP-100 populations, although it was 


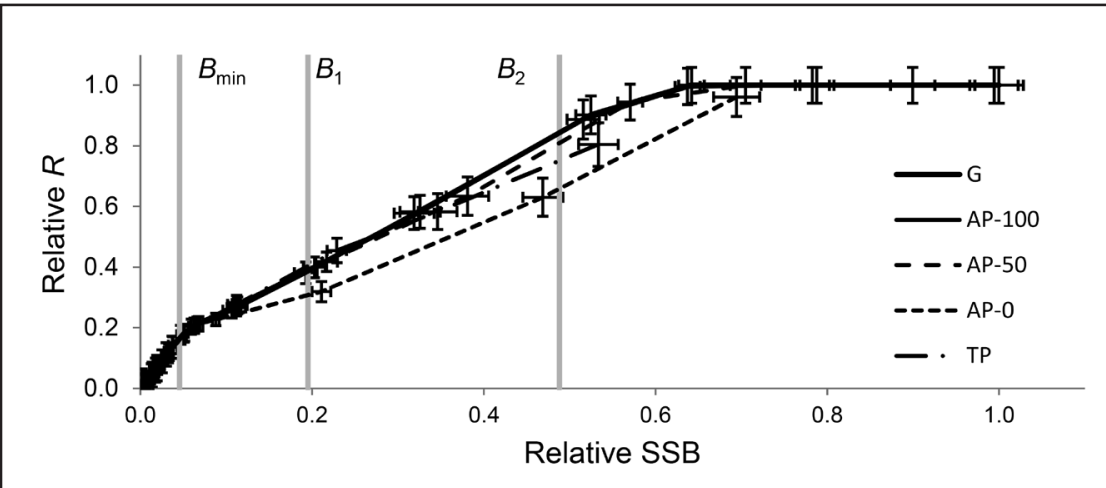

Figure 6

Equilibrium (year 45) relationship between stock and recruitment represented by the relative number of recruits $(R)$ versus the relative spawning stock biomass (SSB) for 5 experimental populations of black sea bass (Centropristis striata): a gonochoristic (G) population (thick, solid line), atypical protogynous (AP) populations with 100\% (AP-100; thin, solid line), 50\% (AP-50; dashed line), and 0\% (AP-0; dotted line) of mature secondary males contributing to spawning, and a typical protogynous population (TP; dashed-dotted line). Each line indicates the average $R$ and SSB (from 100 runs performed at each value of $F$ ) in relation to the average $R$ and SSB of the Gopulation at $F=0.0$; vertical and horizontal bars represent $95 \%$ confidence intervals for relative $R$ and relative SSB, respectively. The gray reference lines indicate the position of the SSB breakpoints $\left(B_{\min }, B_{1}\right.$, and $\left.B_{2}\right)$ that define the different recruitment productivity bins. These results were obtained by using a length-based population model to study the response of these populations to exploitation.

still slightly (about $3 \%$ ) below it. Similarly, a 50\% rate of spawning by secondary males in the TP population brought stock performance closer to that of the AP-50 population; however, levels for the TP population were $12-17 \%$ lower.

\section{Discussion}

We developed a length-based model to simulate the dynamics of a protogynous fish population and examined the resilience of an atypical protogynous population on the basis of the northern stock of black sea bass. The model allowed evaluation of the impact of exploitation on a population's resilience and productivity across a range of life history attributes. The life history of the northern stock of black sea bass is atypical for a protogynous species because the sex ratio at length is not as skewed toward females at small sizes $(<30 \mathrm{~cm})$ nor toward males at large sizes $(>45 \mathrm{~cm})$, as would be expected in a typical protogynous population. Previously published findings (Alonzo and Mangel, 2004; Heppell et al., 2006; Hamilton et al., 2007) indicate that protogynous stocks are more susceptible to overexploitation than are equivalent gonochoristic stocks. Our results support that conclusion, in particular for typical protogynous stocks, which have highly skewed sex ratios. However, we found that atypical protogynous life histories, for which a higher proportion of the small fish are males, may provide added resiliency to exploitation in comparison with typical protogynous species for which all small fish are females and males arise only as a result of subsequent sex change by females. The different transition rates that led to different proportions of females, secondary males, and dominant males in the 2 types of populations resulted in different SSB and SSB sex ratios and ultimately in higher recruitment for the AP population. Moreover, we found that the ability of the AP population to persist under exploitation increases as a function of contribution of secondary males to spawning.

The different response to exploitation by the G, AP, and TP populations can be explained by considering which individuals contribute to SSB because the composition of the spawning stock defines recruitment and ultimately population persistence. In the $\mathrm{G}$ case, we assumed that all mature males and females contributed to spawning, whereas in the protogynous cases, SSB was the sum of females and the subset of dominant males and had a range of possible contributions from secondary males for the AP populations. Size-selective exploitation primarily targeting large individuals decreased SSB, skewed protogynous population spawning stock sex ratios (Fig. 2, B and C), and made the populations more likely than the $\mathrm{G}$ population to experience decreased fertilization because of sperm-limitation. Within the protogynous scenarios, different sex ratios of mature fish also affected the size of SSB and thus 
$R$, particularly if a substantial portion of the mature males were nonspawning secondary males. For example, even in the absence of fishing, the TP population was less productive than the other populations (Fig. 5) because of a smaller SSB that resulted in recruitment occasionally being drawn from the medium recruitment bin, whereas all other populations had SSB high enough to have $R$ drawn consistently from the high productivity bin

Our results indicate that as the contribution to spawning by secondary males in the AP population increased, SSB approached that of the G species and the population became more resilient to exploitation. The addition of secondary males to the spawning stock not only increased SSB but also shifted the sex ratio and lowered the risk to the population of being sperm-limited. In other words, the more secondary males participated in spawning, the more an AP population would perform as if it were gonochoristic because the spawning stock sex ratio approached 1:1. Consequently, the greater the contribution of secondary males, the more resilient the AP population was to fishing. Sensitivity analyses indicated that the same was true for the TP population; however, for equivalent scenarios (i.e., both having $50 \%$ or $100 \%$ of secondary males spawn) the AP population was still more resilient than the TP population, although the magnitude of the difference depended on the proportion of secondary males included in SSB.

The presence of male recruits in the AP population was also an important characteristic. It not only dampened the effect of size-selective exploitation by changing the sex ratios at length but also provided a constant supply of males to the population from a source other than postmaturational sex change. Although transition rates were held constant in our model, previous research (Huntsman and Schaaf, 1994; Alonzo and Mangel, 2004; Taylor, 2013) suggests that sex change is likely to be socially controlled and transition rates may increase in response to removal of males as a way to maintain stable sex ratios. Under severe exploitation, such compensatory sex change would be the only avenue for a typical protogynous population to avoid extremely low abundance of males. In contrast, an atypical protogynous life history, with males available beginning at recruitment, provided an additional buffer against overharvesting of dominant males. Our sensitivity analyses support this interpretation with results indicating that abundance and SSB in an AP configuration were more robust than abundance and SSB in the TP case in response to variations in the rate of sex change. In addition, the proportion of mature dominant males played a significant role because a higher fraction of dominant males brought sex ratios closer to a gonochoristic situation and rendered the population more resilient to fishing.

The life history characteristics of the AP population increased robustness in response to exploitation, and we expect they would also improve resistance to changes in capture selectivity. Results from sensitivity analy- ses on the selection pattern indicated that a reduction in size at selection had some influence on abundance for the AP-50 population. Because any changes to selectivity would apply across all scenarios, we would expect similar results in the other populations if we tested them. However, as seen with other factors, the TP population was generally more vulnerable to a drop in recruitment because of a decrease in SSB or an overly skewed SSB sex ratio; therefore, reducing the size at selectivity would likely affect this population more severely. The sensitivity tests were not performed in a multiplicative manner, and, as a result, it is possible that a response to changes to exploitation with changes to transition rates could alter the outcome. In addition, the effect of dome-shaped selectivity was not explored here because of the lack of empirical evidence supporting it, but the influence of this type of selectivity could be explored in the future.

The sex ratio at maturity, the presence of mature secondary males, and large mature females all worked toward making the AP population more stable than a population with a typical protogynous life history. Black sea bass north of Cape Hatteras undergo seasonal migrations to the edge of the continental shelfmovements that in some cases cover long distances (Musick and Mercer, 1977; Moser and Shepherd, 2009). Such behavior is generally not seen in tropical or subtropical species for which hermaphroditism is most common. The advantages of hermaphroditism evolved in natural systems where there is a selective advantage of size differences between sexes (Ghiselin, 1969; Warner, 1975; Kazancioğlu and Alonzo, 2010).

The selective advantage gained by beginning life as female then switching to male as needed depends on feedback about the existing sex ratio within the reproductive community; too many large males would reduce any reproductive size advantage and only increase sperm competition (Petersen, 1991). However, in a migratory species adopting this life history, the feedback loop may be seasonally interrupted. If individual fish stray from the group with the sex ratio that elicited the sex change, the advantage of being a large male could be lost. In the northern stock of black sea bass, results from a tagging study indicate that the farther the migration, the lower the probability of returning to the original spawning aggregation (Moser and Shepherd, 2009). Consequently, the possibility of returning to a group with large males already present would reduce the advantage gained by switching sex before the migration. Under such conditions, gonochoristic traits may provide more reproductive stability than typical protogyny.

Although our model inputs were based on empirical data as much as possible, we had to make assumptions about several key population processes. The relationship between SSB, spawning stock sex ratio, and subsequent recruitment for black sea bass is poorly understood, and productivity might be highly influenced by environmental factors (Able and Hales, 1997). Given the absence of an accepted stock-recruitment model, 
we used a binned recruitment approach on the basis of empirical data, recognizing that the intent of our model was to evaluate the contrast in response between the population life histories rather than to estimate absolute abundances. We included spawning stock sex ratio as bounds within the stock-recruitment relationship for cases where the sex ratio became extremely skewed.

Even though our approach was rather rudimentary, it allowed us to highlight the importance of incorporating spawning stock sex ratio in addition to SSB in the determination of recruitment for protogynous stocks. If a small proportion of males can successfully fertilize all mature females, the population could remain fully productive even with a skewed sex ratio. In contrast, if the optimal fertilization sex ratio is close to $1: 1$, recruitment would drop if the spawning stock sex ratio became more skewed toward females, as seen in our sensitivity analyses, which showed that recruitment was sensitive to changes in the sex ratio thresholds for the spawning stock. These findings also emphasized the need for more information on spawning behavior of black sea bass and of protogynous hermaphrodite species in general. Specifically, data on male-to-female ratios within a spawning event would help to improve understanding of when a population might be susceptible to sperm limitation or egg limitation and to evaluate the effectiveness of using the combined male and female SSB for populations with skewed sex ratios. Finally, it is unknown to what degree compensatory recruitment may occur and to what extent such recruitment might alter population resilience under exploitation. Given the lack of data to inform this process, we chose not to include it in our model; however it could warrant further exploration.

Transition rates were determined to produce sex ratios at length closely resembling those of the northern stock of black sea bass, but actual transition rates, including their timing and the split between secondary and dominant males, are unknown. We assumed that sex change occurred quickly and that rates of transition from secondary to dominant male were the same for all protogynous populations because our main concerns were to investigate the implication of the atypical life history and the contribution of secondary males to spawning in the north. The duration of the transition made little difference in our model as long as it did not overlap with the spawning period; as long as all transitions were completed before SSB was calculated, there was little influence from this factor. However, it is possible that true transition probabilities differ significantly from what we assumed and that transition rates may differ between an atypical versus typical hermaphrodite population. Moreover, we did not incorporate density dependence into our model, although density-dependent transition rates (compensatory sex change) may have a significant effect on adjusting sex ratios under high fishing pressure (Ellis and Powers, 2012). Molloy et al. (2007) found that flexibility in size at sex change may make protogynous populations as resilient to fishing as gonochoristic populations; there- fore, this feature should be included in future research, if possible.

We did not model social behavior, competition among males, or density-dependent changes in fertilization success for each life stage in part because of a lack of empirical data. The lack of secondary sex characteristics in secondary males would indicate that they are opportunistic spawners rather than direct competitors of dominant males. Although it is unlikely that males participating in spawning as secondary males would be as effective as the dominant males from a behavioral perspective, there is evidence that relative gonad size of secondary males may be larger than that of dominant males, resulting in increased likelihood of fertilization success (Knapp and Carlisle, 2011).

Finally, other possible factors, such as density- or size-dependent $M$, movement, or spatial patterns, were not incorporated into the model. We also assumed that growth was constant across sexes; however, if sexual dimorphic growth favoring males exists, one would expect the G and AP scenarios to become even more aligned.

Despite the limitations of the data and the model, the results of this research provide new insight into the response of protogynous species to exploitation. In particular, atypical sex ratios at length and possible contribution of secondary males to spawning have the potential to significantly increase the resilience of a protogynous stock to fishing. This study indicates that stocks exhibiting atypical protogynous characteristics, such as the northern stock of black sea bass, may be more resilient to exploitation than typical protogynous hermaphrodites characterized by dominant males monopolizing spawning opportunities. In addition, our results highlight the need for a better understanding of factors that govern key processes, such as sex change, secondary male spawning, and recruitment, in protogynous species in general and in black sea bass in particular.

\section{Acknowledgments}

The authors thank A. Seaver for his time programming and building a user interface for the model, E. Brooks for her helpful advice concerning the modeling approach, M. Wuenschel for fruitful discussions about reproduction and life history of black sea bass, J. Evans for help with Python, and E. Robillard and J. Dayton for helping us with questions regarding aging of black sea bass. We also are grateful to our reviewers for their constructive comments.

\section{Literature cited}

Able, K. W, and L. S. Hales Jr.

1997. Movements of juvenile black sea bass Centropristis striata (Linnaeus) in a southern New Jersey estuary. J. Exp. Mar. Biol. Ecol. 213:153-167. 
Allsop, D. J., and S. A. West. 2004. Sex-ratio evolution in sex changing animals. Evolution 58:1019-1027. Article

Alonzo, S. H., and M. Mangel.

2004. The effects of size-selective fisheries on the stock dynamics of and sperm-limitation in sex-changing fish. Fish. Bull. 102:1-13.

Alonzo, S. H., T. Ish, M. Key, A. D. MacCall, and M. Mangel. 2008. The importance of incorporating protogynous sex change into stock assessments. Bull. Mar. Sci. 83:163179.

Bowen, B. W., and J. C. Avise.

1990. Genetic structure of Atlantic and Gulf of Mexico populations of sea bass, menhaden, and sturgeon: influence of zoogeographic factors and life-history patterns. Mar. Biol. 107:371-381. Article

Brander, K.

2003. What kinds of fish stock predictions do we need and what kinds of information will help us to make better predictions? Sci. Mar. 67 (suppl. 1):21-33.

Brooks, E. N., K. W. Shertzer, T. Gedamke, and D. S. Vaughan. 2007. Stock assessment of protogynous fish: evaluating measures of spawning biomass used to estimate biological reference points. Fish. Bull. 106:12-23.

Collette, B. B., and G. Klein-MacPhee.

2002. Bigelow and Schroeder's fishes of the Gulf of Maine, $3^{\text {rd }}$ ed., 748 p. Smithsonian Institution Press, Washington, DC.

Drohan, A. F., J. P. Manderson, and D. B. Packer.

2007. Essential fish habitat source document: black sea bass, Centropristis striata, life history and habitat characteristics, $2^{\text {nd }}$ ed. NOAA Tech. Memo. NMFS-NE-200, $68 \mathrm{p}$.

Ellis, R. D., and J. E. Powers.

2012. Gag grouper, marine reserves, and density-dependent sex change in the Gulf of Mexico. Fish. Res 115-116:89-98. Article

Ghiselin, M. T.

1969. The evolution of hermaphroditism among animals. Q. Rev. Biol. 44:189-208. Article

Haddon, M.

2001. Modelling and quantitative methods in fisheries, 424 p. Chapman \& Hall/CRC Press, Boca Raton, FL.

Hamilton, S. L., J. E. Caselle, J. D. Standish, D. M. Schroeder, M. S. Love, J. A. Rosales-Casian, and O. Sosa-Nishizaki.

2007. Size-selective harvesting alters life histories of a temperate sex-changing fish. Ecol. Appl. 17:2268-2280. Article

Heppell, S. S., S. A. Heppell, F. C. Coleman, and C. C. Koenig. 2006. Models to compare management options for a protogynous fish. Ecol. Appl. 16:238-249. Article

Huntsman, G. R, and W. E. Schaaf.

1994. Simulation of the impact of fishing on reproduction of a protogynous grouper, the graysby. North Am. J. Fish. Manage. 14:41-52. Article

Kazancioğlu, E., and S. H. Alonzo.

2010. A comparative analysis of sex change in Labridae supports the size advantage hypothesis. Evolution 64:2254-2264. Article

Knapp, R., and S. L. Carlisle.

2011. Testicular function and hormonal regulation in fishes. In Hormones and reproduction of vertebrates, vol. 1: fish- es (D. O. Norris and K. H. Lopez, eds.), p. 43-63. Academic Press, Burlington, MA. Article

Lavenda, N.

1949. Sexual difference and normal protogynous hermaphroditism in the Atlantic sea bass, Centropristes striatus. Copeia 3:185-194. Article

McCartney, M. A., M. L. Burton, and T. G. Lima.

2013. Mitochondrial DNA differentiation between populations of black sea bass (Centropristis striata) across Cape Hatteras, North Carolina (USA). J. Biogeogr. 40:1386-1398. Article

Molloy, P. P., N. B. Goodwin, I. M. Côté, M. J. G. Gage, and J. D. Reynolds.

2007. Predicting the effects of exploitation on male-first sex-changing fish. Anim. Conserv. 10:30-38. Article

Moser, J., and G. R. Shepherd.

2009. Seasonal distribution and movement of black sea bass (Centropristis striata) in the northwest Atlantic as determined from a mark-recapture experiment. J. Northwest Atl. Fish. Sci. 40:17-28. Article

Munday, P. L., P. M. Buston, and R. R. Warner.

2006. Diversity and flexibility of sex-change strategies in animals. Trends Ecol. Evol. 21:89-95. Article

Musick, J. A., and L. P. Mercer.

1977. Seasonal distribution of black sea bass, Centropris tis striata, in the Mid-Atlantic Bight with comments on the ecology and fisheries of the species. Trans. Am. Fish. Soc. 106:12-25. Article

Petersen, C. W.

1991. Sex allocation in hermaphroditic sea basses. Am Nat. 138:650-667. Article

Pitcher, T. J. (ed.)

1993. Behaviour of teleost fishes, $2^{\text {nd }}$ ed., 740 p. Chapman \& Hall, London.

Pitcher, T. J., and P. D. M. MacDonald.

1973. Two models for seasonal growth in fishes. J. Appl. Ecol. 10:599-606. Article

Quinn, T. J., II, and R. B. Deriso.

1999. Quantitative fish dynamics, 560 p. Oxford Univ. Press, New York.

Roy, E. M, J. M. Quattro, and T. W. Greig.

2012. Genetic management of black sea bass: influence of biogeographic barriers on population structure. Mar. Coast. Fish. 4:391-402. Article

Shepherd, G. R., and M. Terceiro.

1994. The summer flounder, scup, and black sea bass fishery of the Middle Atlantic Bight and Southern New England waters. NOAA Tech. Rep. NMFS-122, 13 p.

Taborsky, M.

1994. Sneakers, satellites, and helpers: parasitic and cooperative behavior in fish reproduction. Adv. Study Behav. 23:1-100. Article

Taylor, B. M.

2013. Drivers of protogynous sex change differ across spatial scales. Proc. R. Soc., B 281:20132423. Article

Warner, R. R.

1975. The adaptive significance of sequential hermaphroditism in animals. Am. Nat. 109:61-82. Article

Young, B., D. V. Conti, and M. D. Dean.

2013. Sneaker "jack" males outcompete dominant "hooknose" males under sperm competition in Chinook salmon (Oncorhynchus tshawytscha). Ecol. Evol. 3:4987-4997. Article 